\title{
BMJ Open A cohort study of influences, health outcomes and costs of patients' health-seeking behaviour for minor ailments from primary and emergency care settings
}

\author{
M C Watson, ${ }^{1} \mathrm{~J}$ Ferguson, ${ }^{2} \mathrm{G}$ R Barton, ${ }^{3} \mathrm{~V}$ Maskrey, ${ }^{4}$ A Blyth, ${ }^{4} \mathrm{~V}$ Paudyal, ${ }^{1}$ \\ C M Bond, ${ }^{1}$ R Holland, ${ }^{4}$ T Porteous, ${ }^{1}$ T H Sach, ${ }^{3}$ D Wright, ${ }^{5}$ S Fielding ${ }^{1}$
}

To cite: Watson MC,

Ferguson J, Barton GR, et al. A cohort study of influences, health outcomes and costs of patients' health-seeking behaviour for minor ailments from primary and emergency care settings. BMJ Open 2015;5:e006261.

doi:10.1136/bmjopen-2014006261

- Prepublication history and additional material is available. To view please visit the journal (http://dx.doi.org/ 10.1136/bmjopen-2014006261).

Received 17 September 2014 Revised 22 December 2014 Accepted 30 December 2014

CrossMark

For numbered affiliations see end of article.

Correspondence to Dr M C Watson; m.c.watson@abdn.ac.uk

\section{ABSTRACT}

Objectives: To compare health-related and cost-related outcomes of consultations for symptoms suggestive of minor ailments in emergency departments (EDs), general practices and community pharmacies.

Design: Observational study; prospective cohort design.

Setting: EDs $(n=2)$, general practices $(n=6)$ and community pharmacies $(n=10)$ in a mix of rural/urban and deprived/affluent areas across North East Scotland and East Anglia. Participants Adults ( $\geq 18$ years) presenting between 09:00 and 18:00 (Monday-Friday) in general practices and 09:00-18:00 (MondaySaturday) in pharmacies and EDs with $\geq 1$ of the following: musculoskeletal pain; eye discomfort; gastrointestinal disturbance; or upper respiratory tract-related symptoms.

Interventions: Participants completed three questionnaires: baseline (prior to index consultation); satisfaction with index consultation and follow-up (2 weeks after index consultation).

Main outcome measures: Symptom resolution, quality of life, costs, satisfaction and influences on care-seeking behaviour.

Results: 377 patients participated, recruited from EDs (81), general practices (162) and community pharmacies (134). The 2-week response rate was 70\% (264/377). Symptom resolution was similar across all three settings: $\mathrm{ED}(37.3 \%)$, general practice $(35.7 \%)$ and pharmacy (44.3\%). Mean overall costs per consultation were significantly lower for pharmacy (£29.30 (95\% Cl £21.60 to £37.00)) compared with general practice (£82.34 (95\% Cl £63.10 to £101.58)) and ED (£147.09 (95\% Cl £125.32 to £168.85)). Satisfaction varied across settings and by measure used. Compared with pharmacy and general practice use, ED use was significantly $(p<0.001)$ associated with first episode and short duration of symptom(s), as well as higher levels of perceived seriousness and urgency for seeking care. Convenience of location was the most common reason for choice of consultation setting.

Conclusions: These results suggest similar healthrelated outcomes and substantially lower costs with

\section{Strengths and limitations of this study}

- This is the first study to compare health-related and cost-related outcomes across the three main healthcare settings where patients present with symptoms suggestive of minor ailments.

- Prospective data collection using the same processes and tools across all three settings reduces the risk of bias.

- While the inclusion of different types of pharmacies and a variety of locations of pharmacies and general practices increased the generalisability of the results, more rural communities were under-represented.

- A cohort study design was used rather than a randomised controlled trial because the latter was an impractical option to address the objectives of this study.

pharmacy consultations for minor ailments. Effective strategies are now needed to shift demand for minor ailment management away from EDs and general practices to the community pharmacy setting.

\section{INTRODUCTION}

Emergency departments (EDs) are reported to be in crisis ${ }^{1}$ with 22 million attendances in EDs in England between 2012 and 2013, one third of which involved 'guidance or advice only'. This is placing 'intense, growing and unsustainable pressure' on the service. ${ }^{3}$ In general practice, 340 million general practice consultations occurred between 2012 and 2013. ${ }^{3}$ This equates to an estimated 5.5 consultations per patient annually; $40 \%$ higher than 1995. ${ }^{4}$ Recent estimates suggest that $5 \%$ and $13 \%$ of consultations in ED and general practice, respectively, are for minor ailments that could be managed in community pharmacies. ${ }^{5}$ 
For the purpose of this study, minor ailments are "common or self-limiting or uncomplicated conditions which may be diagnosed and managed without medical (ie, doctor) intervention., ${ }^{6}$

The above illustrates that health seeking for the management of minor ailments from high-cost settings such as EDs and general practice persists despite a wide range of alternative sources of care being widely available. Effective over-the-counter (OTC) medicines can be supplied from community pharmacies to support the management of minor ailments. ${ }^{7}$ Over the past 30 years, many medicines have been reclassified from "prescription only' status to OTC medicines and this has increased the options available for the treatment of minor ailments. Local and national pharmacy-based minor ailment schemes have also been introduced to reduce demand on high-cost settings, with evidence from a recent systematic review suggesting that they appear to be effective. ${ }^{8}$ Pharmacy-based minor ailment schemes provide public access to NHS treatment and/or advice via a pharmacist or pharmacy personnel, or, where appropriate, to onward referral to other health professionals. These schemes were originally proposed by the UK health departments as part of their long-term strategy to encourage patient self-care and utilisation of pharmacies as the first port of call for minor ailments where professional support was required. However, the evidence to date is limited in terms of study quality and outcomes reported, that is, generally surrogate health outcomes have been evaluated, such as reconsultation rates. Furthermore, no formal economic evaluations have been reported.

There is an urgent need to shift demand for minor ailment management from high-cost settings to potentially cheaper alternatives, for example, community pharmacies. Reassurance, however, is needed that similar outcomes can be achieved with community pharmacy management compared with these other settings. The purpose of this study was to compare health-related and cost-related outcomes associated with the management of symptoms suggestive of minor ailments (hereafter referred to as minor ailments) in three healthcare settings: ED, general practice and community pharmacy. The following objectives were assessed:

1. Whether health-related and cost-related outcomes differ between settings.

2. Whether satisfaction with index consultation is associated with health-related outcomes.

3. What factors (triggers) influence patients' choice of care setting.

\section{METHODS}

Study design

A prospective cohort study was conducted.

\section{Study setting and recruitment}

The study was conducted across two geographic regions (East Anglia, England and Grampian, Scotland).
Potential participating sites were identified using NHS lists of health service providers. General practices and community pharmacies were eligible if they were within a 25-mile radius of the main city in East Anglia (Norwich) and Grampian (Aberdeen). This was to ensure that participants could realistically have chosen from all three settings of interest. In Grampian, a letter of invitation was sent to all eligible practices and pharmacies. In East Anglia, the same approach was used for pharmacies. From those expressing an interest (online supplementary appendix 1), purposive sampling was used to generate a maximum variation sample to include different types and locations of pharmacies and different locations of general practices. Three general practices were purposively selected in East Anglia to achieve maximum variation.

\section{Participant recruitment}

Participant recruitment was undertaken by six researchers between July 2012 and April 2013. The same process for data collection was used across all settings. Participants were eligible for inclusion if they: were aged $\geq 18$ years; requested treatment for one or more of the four included minor ailments or presented symptoms associated with these ailments; requested medicines for the treatment of one of the four minor ailments (pharmacy only); presented between 09:00 and 18:00, Monday-Saturday (ED and pharmacy) and between 09:00 and 18:00, Monday-Friday (general practice); had a face-to-face consultation (ie, interaction with member of staff). This consultation is referred to hereafter as the index consultation'. These recruitment periods were selected to reflect the traditional opening hours of community pharmacies (ie, to reflect periods when patients had the option of seeking care from community pharmacies if they had chosen to do so). Patients in pharmacies were excluded if they were collecting prescription items for target minor ailments or purchasing treatment for the future management of those ailments. Patients in any setting were excluded if they did not adequately understand verbal/ written information in English. The same recruitment method was used across all sites and settings. Promotional posters were displayed throughout participating sites. Receptionists (ED and general practice) and pharmacy staff could also direct potential participants to the researchers who were based within each premises. The researchers were not permitted to approach patients directly due to the NHS Ethics Committee's decision. The researchers applied the inclusion criteria and sought informed consent from all eligible and willing patients.

The four included symptoms/ailments were:

- Musculoskeletal pain: aches or pain in arms or legs or back or hands or feet;

- Eye discomfort;

- Gastrointestinal disturbance: nausea or vomiting or diarrhoea or constipation;

- Upper respiratory tract-related: sore throat or cough or cold or sinus problems. 
The above groups of symptoms were chosen because they have previously been identified as occurring frequently within EDs and general practices. ${ }^{5}$

\section{Data collection}

Data collection was undertaken using the same process across all settings to minimise bias. Participants were asked to self-complete three questionnaires; one at baseline prior to the consultation, one immediately after the consultation and one two weeks later. The content of the questionnaires was informed by the literature and also comprised a range of validated (EQ-5D/EQ-VAS (visual analogue scale), ${ }^{9}$ MISS-21 (Medical Interview satisfaction Scale),${ }^{10}$ triggers for seeking care $^{11}$ ) and non-validated measures (global satisfaction very satisfied/ dissatisfied (5-point)). The MISS-2 ${ }^{10}$ was developed to use with consultations in general practice. The items were rephrased to ensure face validity for their use with participants recruited from the EDs and community pharmacies. The scale comprises four subscales: distress relief; communication; rapport and compliance intent. The urgency for seeking care and perceived severity items were derived from previous research. ${ }^{12} 13$

The purpose of the baseline questionnaire was to collect basic demographics, triggers for seeking care and contact details for follow-up. Baseline information was also collected on potential predictors or confounders and included the index minor ailment, age, gender, employment status, marital status, living alone, whether it was the first time they had experienced the symptoms, how serious the symptoms were perceived to be, belief on timing of visit, how long they had had the ailment, and baseline EQ-5D visual analogue scale (EQ-VAS).

The postconsultation questionnaire asked about satisfaction (global satisfaction and MISS-21), the outcome of the consultation (eg, medicines sold or supplied) and associated costs. The 2-week follow-up questionnaire asked participants about symptom resolution, quality of life, re-consultation for the index ailment and health service utilisation since their index consultation.

One version was used for each setting (ED, general practice, community pharmacy) and differed only in sitespecific questions relevant to the triggers for seeking care. Although developed for self-completion, if asked to do so, a researcher would assist participants in completing the baseline and/or satisfaction questionnaires by reading the questions and response options to the participant. Participants were permitted to take the postconsultation questionnaire away with them (with a reply-paid envelope) for later completion if necessary. Follow-up questionnaires were mailed to participants. They were given the option of self-completing these and returning them by mail or completing it with a researcher over the phone. Those opting for mailed questionnaires were sent reminders at 2 and 4 weeks after the initial mailing. The study processes and documentation (questionnaires) were piloted over a 3.5-day period and included 28 patients recruited from one pharmacy $(n=3)$, one general practice $(n=11)$ and the ED $(n=14)$ in Grampian. Changes were made to the promotional materials and questionnaires as a result of piloting.

\section{Exposure and outcomes}

The exposure of interest was setting of care: ED, general practice or pharmacy. The primary outcome was symptom resolution 2 weeks after the index consultation. Secondary outcomes (collected in the postconsultation questionnaire) were: sold or supplied medicines, satisfaction measured by MISS-21, ${ }^{10}$ participant experience, duration of consultation and quality of life (QoL) measured by the EQ-5D-3L. ${ }^{14}$ Triggers for choice of site of presentation are reported as a secondary outcome from the baseline questionnaire.

\section{Sample size}

A symptom resolution rate of $70 \%$ was assumed based on a previous estimate. ${ }^{15} \mathrm{~A}$ minimum of 81 participants were required per setting. The prevalence of minor ailments was anticipated to be higher in community pharmacies and general practices ${ }^{5}$ compared with EDs. As such, recruitment targets for the higher prevalence sites were set at 160 participants and from the EDs were 80 . Based on these estimates, the study would have over $80 \%$ power at a $5 \%$ level to detect differences of $10 \%$ between symptom resolution rates of $70 \%$ (95\% CI $60 \%$ to $80 \%$ ) across settings.

\section{Statistical methods and analysis}

Data were entered into an IBM SPSS Statistics V.20 database and a $10 \%$ random sample checked for accuracy. Categorical variables were described with frequency and valid percentages. Normally distributed continuous variables were summarised using mean and SD with median and IQR provided if data were skewed. Comparison of categorical variables between sites of presentation (ED, general practice, pharmacy) was undertaken using $\chi^{2}$ tests or Fisher's exact test where required. Comparison of continuous variables between sites was undertaken using analysis of variance (ANOVA) if normally distributed and Kruskal-Wallis if skewed. Logistic regression was used to ascertain predictors of binary outcomes (eg, symptom resolution). Linear regression was used to model QoL (EQ-5D) at follow-up. Variables were included in these models if significant $(\mathrm{p} \leq 0.05)$ in the univariate analysis (independent t-test, ANOVA, correlation as appropriate) or belonged to an a priori list of variables for inclusion (site, ailment, age, gender, baseline EQ-5D and duration of symptoms). All analyses were undertaken on complete cases for that outcome.

For each participant, costs were estimated for the index consultation and any actions taken in the next 2 weeks for the original minor ailment in question (health professional contacts, medication and investigations). Both NHS and any patient out of pocket costs (for medication only) were included. Costs were estimated in UK sterling (£) at 2011/2012 financial year 
levels, but not discounted as the time frame was less than 1 year. A complete case approach ${ }^{16}$ was adopted at each time point. Thus, costs were only estimated if data were available for each aspect of resource use for the index consultation/follow-up questionnaire. Cost per participant was estimated by summing the index consultation cost and the costs incurred in the 2 weeks thereafter. To estimate the impact of the index consultation on health-related QoL, participants were asked to complete the EuroQoL EQ-5D-3L ${ }^{14}$ at baseline and 2-week follow-up. This enabled a cost-utility analysis to be undertaken. Quality adjusted life year (QALY) scores were only calculated for those with complete baseline and follow-up EQ-5D-3L scores, and it was assumed scores changed linearly between these two time points. ${ }^{17}$

STATA V.11 was used for all economic analyses; $p$ values $\leq 0.05$ were deemed statistically significant. Multiple linear regression ${ }^{17}$ with backward stepwise selection was used to estimate the mean cost difference (incremental cost) between the three settings and the mean QALY difference (incremental effect). Individuals with missing data for any of the covariates in the final model were excluded. If care in one setting did not dominate the care provided in the other two settings, ${ }^{18}$ the incremental cost per QALY gain (incremental cost-effectiveness ratio (ICER)) was calculated (mean incremental cost/mean incremental QALY gain) by comparing each remaining setting with the next cheapest. In line with National Institute for Health and Care Excellence (NICE) guidance, ${ }^{19}$ any calculated ICER would be compared with a cost-effectiveness threshold $(\lambda)$ of $£ 20000$ per QALY.

\section{Subgroup analysis}

Resolution of symptoms was compared separately across sites for each minor ailment (where numbers permitted). QoL outcomes (EQ-5D-3L utility and EQ-VAS), participant experience and MISS-21 scales at baseline and follow-up were compared separately within site for individuals whose symptoms did and did not resolve.

\section{RESULTS}

\section{Setting characteristics}

Two EDs, 6 general practices and 10 community pharmacies participated, equally distributed across the two regions. Both EDs were located in teaching hospitals. Of the 10 pharmacies, 6 were large chain, 2 small-chain and 2 independent single outlets. Four general practices were in urban areas. The pharmacies and practices were located across a range of deprivation codes (online supplementary appendix 1).

\section{Participant characteristics}

In total 377 participants were recruited, $94.3 \%$ of the target sample size $(n=400$; table 1$)$. Of these, 81,162 and 134 were recruited from EDs, general practices and community pharmacies, respectively. Thus target recruitment was met for general practice and ED. Participant characteristics by setting are shown in table 1. Participants recruited from community pharmacies were significantly older than those recruited elsewhere. ED participants were significantly younger and more likely to be single compared with participants recruited in general practices and pharmacies.

Most participants presented with musculoskeletal aches and pains $(46.4 \%, \mathrm{n}=175)$ or upper respiratory tract symptoms $(28.9 \%, \mathrm{n}=109)$ ). Presenting condition varied across settings, for example, musculoskeletal symptoms dominated in ED (81.5\%, $\mathrm{n}=66$; table 1). In total, 39.2\% $(\mathrm{n}=147)$ of participants were experiencing the symptom for the first time $(64.2 \% \quad(n=52)$ in ED, 38.8\% ( $n=62)$ in general practices and $24.6 \%(\mathrm{n}=33)$ in pharmacies $)$. Participants recruited from ED were significantly more likely to have had the symptom for a short period of time prior to their index consultation (median 1 day (IQR 0.5-7.0)) compared with participants in general practice (median 14 days (IQR 3-42)). ED participants were significantly more likely to consider their symptom to be 'somewhat serious' compared with participants recruited from other sites and were significantly more likely $(67.5 \%,(\mathrm{n}=52))$ to state that they needed to seek care for their symptom(s) within $24 \mathrm{~h}$ compared with general practice $(47.2 \%,(\mathrm{n}=76))$ and pharmacy-based participants $(37.9 \%,(\mathrm{n}=50))$.

Data from postconsultation questionnaires were available for $89.7 \%$ (338/377) of participants: ED (77.8\%, $63 / 81)$, general practice $(87 \%, 141 / 162)$ and pharmacy $(100 \%)$. The follow-up questionnaire was completed by $263(69.8 \%)$ participants: 63\% (51/81) ED, $71.6 \%$ (116/162) general practice and 72\% (97/134) pharmacy. The response rates did not differ significantly across the settings $(\mathrm{p}=0.29)$.

\section{Health-related outcomes}

\section{Primary outcome: symptom resolution}

Median follow-up was 17 days (IQR 15-21) and most (86.1\%) participants were followed up within 1 month. At follow-up, the proportion of symptoms that had resolved was similar across settings with $37.3 \%$ resolving in $\mathrm{ED}, 35.7 \%$ in general practice and $44.3 \%$ in pharmacy ( $p=0.415$, table 2$)$. Univariate analysis showed that significant predictors of symptom resolution were: the type of presenting symptom, its perceived seriousness, the urgency with which the participant believed they should seek help, duration of symptoms and baseline QoL (table 3). In the adjusted model, significant predictors were: presenting symptom and belief about the urgency with which they should visit their chosen site (ie, 24 or $>24 \mathrm{~h}$; table 3). Participants who believed they needed to consult about their symptoms within $24 \mathrm{~h}$ were more likely to report symptom resolution. The odds of resolution were significantly higher for non-musculoskeletal symptoms. Setting was not a significant predictor of resolution.

\section{Secondary outcomes: postconsultation}

A significantly higher proportion $(\mathrm{p}<0.001)$ of participants was sold, prescribed or supplied medicines from 
Table 1 Characteristics of participants by site including information on the symptoms per cent $(n)$

\begin{tabular}{|c|c|c|c|c|c|}
\hline & $\begin{array}{l}\text { Overall } \\
(\mathrm{N}=377)\end{array}$ & ED (N=81) & $\begin{array}{l}\text { General } \\
\text { practice } \\
(\mathrm{N}=162)\end{array}$ & $\begin{array}{l}\text { Pharmacy } \\
(\mathrm{N}=134)\end{array}$ & p Value* \\
\hline \multicolumn{6}{|l|}{ Participant demographics } \\
\hline \multicolumn{6}{|l|}{ Age } \\
\hline Mean (SD) & $48.3(17.8)$ & $42.2(17.9)$ & $48.3(17.6)$ & $51.9(17.1)$ & $0.001 \dagger$ \\
\hline Missing & (7) & (4) & (2) & (1) & \\
\hline \multicolumn{6}{|l|}{ Gender } \\
\hline Male & $40.6(151)$ & $44.9(35)$ & $40.4(65)$ & $38.3(51)$ & $0.646 \ddagger$ \\
\hline Female & $59.4(221)$ & $55.1(43)$ & $59.6(96)$ & $61.7(82)$ & \\
\hline Missing & $(5)$ & (3) & (1) & (1) & \\
\hline \multicolumn{6}{|l|}{ Employment } \\
\hline Employed full-time & $60.5(225)$ & $66.7(52)$ & $64.6(104)$ & $51.9(69)$ & $0.006 \ddagger$ \\
\hline Retired & $22.0(82)$ & $10.3(8)$ & $23.6(38)$ & $27.1(36)$ & \\
\hline $\begin{array}{l}\text { Other (including student, } \\
\text { unemployed) }\end{array}$ & $17.5(65)$ & $23.1(18)$ & $11.8(19)$ & $21.1(28)$ & \\
\hline Missing & (5) & (3) & (1) & (1) & \\
\hline \multicolumn{6}{|l|}{ Registered MAS§ } \\
\hline Yes & $10.2(38)$ & $10.4(8)$ & $6.2(10)$ & $15.0(20)$ & $0.079 \ddagger$ \\
\hline No & $81.1(301)$ & $84.4(65)$ & $82.6(133)$ & $77.4(103)$ & \\
\hline Do not know & $8.6(32)$ & $5.2(4)$ & $11.2(18)$ & $7.5(10)$ & \\
\hline Missing & (6) & (4) & (1) & (1) & \\
\hline \multicolumn{6}{|l|}{ Marital Status } \\
\hline Single & $24.2(90)$ & $37.2(29)$ & $22.4(36)$ & $18.8(25)$ & $0.012 \ddagger$ \\
\hline Married/living with partner & $61.6(229)$ & $53.8(42)$ & $65.2(105)$ & $61.7(82)$ & \\
\hline Divorced/separated/widowed & $14.2(53)$ & $9.0(7)$ & $12.4(20)$ & $19.5(26)$ & \\
\hline Missing & $(5)$ & (3) & (1) & $(1)$ & \\
\hline \multicolumn{6}{|l|}{ Live alone } \\
\hline Yes & $20.3(75)$ & $13.2(10)$ & $18.8(30)$ & $26.3(35)$ & $0.061 \neq$ \\
\hline No & $79.7(294)$ & $86.8(66)$ & $81.2(130)$ & 73.7 (98) & \\
\hline Missing & (8) & $(5)$ & $(2)$ & (1) & \\
\hline \multicolumn{6}{|l|}{ Registered with GP in the UK } \\
\hline Yes & $97.8(364)$ & $98.7(77)$ & $98.1(158)$ & $97.0(129)$ & - \\
\hline No & $2.2(8)$ & $1.3(1)$ & $1.9(3)$ & $3.0(4)$ & \\
\hline Missing & (5) & (3) & (1) & (1) & \\
\hline \multicolumn{6}{|l|}{ Nationality } \\
\hline British & $94.9(351)$ & $93.6(73)$ & $96.2(153)$ & $94.0(125)$ & - \\
\hline Other EU & $3.0(11)$ & $2.6(2)$ & $3.1(5)$ & $3.0(4)$ & \\
\hline Non-EU & $2.2(8)$ & $3.8(3)$ & $0.6(1)$ & $3.0(4)$ & \\
\hline Missing & $(7)$ & (3) & (3) & (1) & \\
\hline Norfolk only & $(\mathrm{N}=163)$ & $(\mathrm{N}=35)$ & $(\mathrm{N}=71)$ & $(\mathrm{N}=57)$ & \\
\hline \multicolumn{6}{|l|}{ Pay for prescriptions } \\
\hline Yes & $51.2(82)$ & $57.6(19)$ & $50.0(35)$ & $49.1(28)$ & $0.713 \ddagger$ \\
\hline No & $48.8(78)$ & $42.4(14)$ & $50.0(35)$ & $50.9(29)$ & \\
\hline Do not know/missing ${ }^{*}$ & (3) & $(2)$ & (1) & (0) & \\
\hline \multicolumn{6}{|l|}{ Ailment characteristics } \\
\hline \multicolumn{6}{|l|}{ Presenting Symptoms } \\
\hline Musculoskeletal aches and pains & $46.4(175)$ & $81.5(66)$ & $36.4(59)$ & $37.3(50)$ & $<0.001 \ddagger$ \\
\hline Eye-related & $11.4(43)$ & $14.8(12)$ & $11.1(18)$ & $9.7(13)$ & \\
\hline Stomach upset & $4.8(18)$ & $1.2(1)$ & $6.2(10)$ & $5.2(7)$ & \\
\hline Upper respiratory tract & $28.9(109)$ & $0(0)$ & $34.0(55)$ & $40.3(54)$ & \\
\hline Multiple symptoms & $8.5(32)$ & $2.5(2)$ & $12.3(20)$ & $7.5(10)$ & \\
\hline \multicolumn{6}{|l|}{ First time had symptoms } \\
\hline Yes & $39.2(147)$ & $64.2(52)$ & $38.8(62)$ & $24.6(33)$ & $<0.001 \ddagger$ \\
\hline No & $60.8(230)$ & $35.8(29)$ & $61.3(98)$ & $75.4(101)$ & \\
\hline \multirow[t]{2}{*}{ Missing } & (2) & (0) & (2) & $(0)$ & \\
\hline & & & & & Continued \\
\hline
\end{tabular}


Table 1 Continued

\begin{tabular}{|c|c|c|c|c|c|}
\hline & $\begin{array}{l}\text { Overall } \\
(\mathrm{N}=377)\end{array}$ & ED $(N=81)$ & $\begin{array}{l}\text { General } \\
\text { practice } \\
(\mathrm{N}=162)\end{array}$ & $\begin{array}{l}\text { Pharmacy } \\
(\mathrm{N}=134)\end{array}$ & p Value* \\
\hline \multicolumn{6}{|c|}{ How serious are the symptoms? } \\
\hline Not serious at all & $37.0(136)$ & $16.9(13)$ & $27.5(43)$ & $60.2(80)$ & \multirow[t]{4}{*}{$<0.001 \ddagger$} \\
\hline Somewhat serious & $54.1(199)$ & $75.3(58)$ & $61.4(97)$ & $33.1(44)$ & \\
\hline Very serious & $9.0(33)$ & $7.8(6)$ & $11.4(18)$ & $6.8(9)$ & \\
\hline Missing & (9) & (4) & (4) & (1) & \\
\hline \multicolumn{6}{|c|}{ How soon did you need to visit? } \\
\hline Within $24 \mathrm{~h}$ & $48.1(178)$ & $67.5(52)$ & $47.2(76)$ & $37.9(50)$ & \multirow[t]{3}{*}{$<0.001 \ddagger$} \\
\hline $24 \mathrm{~h}$ or more & 51.9 (192) & $32.5(25)$ & $52.8(85)$ & $62.1(82)$ & \\
\hline Missing & (7) & (4) & (1) & (2) & \\
\hline \multicolumn{6}{|l|}{ Duration of symptoms } \\
\hline Median (IQR) in days & $7(2-35)$ & $1.0(0.5-7.0)$ & $14(3-42)$ & $7(2-80)$ & \multirow{3}{*}{$\begin{array}{r}<0.0019 \\
0.455 \dagger\end{array}$} \\
\hline Baseline EQ-VAS & $(n=370)$ & $(n=76)$ & $(n=160)$ & $(n=134)$ & \\
\hline Mean (SD) & $68.0(19.6)$ & $68.9(22.4)$ & 66.6 (18.9) & $69.3(18.8)$ & \\
\hline $\begin{array}{l}\text { "Tests exclude missing data. } \\
\text { †ANOVA. } \\
\text { †Pearson } \chi^{2} \text {. } \\
\text { §National MAS (Scotland). } \\
\text { qKruskal-Wallis test. } \\
\text { ANOVA, analysis of variance } \\
\text { visual analogue scale. }\end{array}$ & 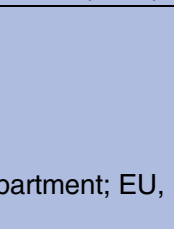 & 1 & 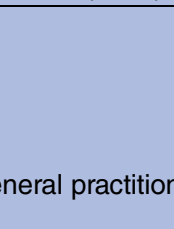 & lingr $A$ & e; VAS, \\
\hline
\end{tabular}

Table 2 Outcomes: resolution of symptoms, quality of life and outcome of index consultation by setting

\begin{tabular}{|c|c|c|c|c|c|}
\hline & Overall \% (n) & ED \% (n) & $\begin{array}{l}\text { General } \\
\text { practice \% (n) }\end{array}$ & Pharmacy \% (n) & p Value \\
\hline Resolution of symptoms at 2 -week follow-up & $(n=263)$ & $(n=51)$ & $(n=116)$ & $(n=97)$ & $0.415^{\star}$ \\
\hline Resolved & $39.2(103)$ & $37.3(19)$ & $35.7(41)$ & $44.3(43)$ & \\
\hline Follow-up EQ-VAS & $(n=261)$ & $(n=50)$ & $(n=115)$ & $(n=96)$ & $0.054 \dagger$ \\
\hline Mean (SD) & $77.4(18.2)$ & $74.8(19.6)$ & $75.5(19.5)$ & $80.9(15.3)$ & \\
\hline Change in EQ-VAS & $(n=259)$ & $(n=50)$ & $(n=113)$ & $(n=96)$ & $0.726 \dagger$ \\
\hline Mean (SD) & $8.43(18.2)$ & $7.6(17.9)$ & $7.8(18.1)$ & $9.6(18.5)$ & \\
\hline Outcome of index consultation & $(n=338)$ & $(n=63)$ & $(n=141)$ & $(n=134)$ & \\
\hline Sold or supplied medicines & $(n=334)$ & $(n=61)$ & $(n=141)$ & $(n=132)$ & $<0.001^{*}$ \\
\hline Yes & $66.5(222)$ & $31.1(19)$ & $61.7(87)$ & $87.9(116)$ & \\
\hline Sold or supplied items & $(n=327)$ & $(n=59)$ & $(n=138)$ & $(n=130)$ & $<0.001^{*}$ \\
\hline Yes & $9.3(35)$ & $37.3(22)$ & $2.2(3)$ & $7.7(10)$ & \\
\hline Satisfaction & $(n=334)$ & $(n=60)$ & $(n=136)$ & $(n=127)$ & $0.115^{\star} \ddagger$ \\
\hline Satisfied & 64.7 (209) & $53.3(32)$ & $66.2(90)$ & $68.5(87)$ & \\
\hline Neither & 31.9 (103) & $45.0(27)$ & $27.9(38)$ & $29.9(38)$ & \\
\hline Dissatisfied & $3.4(11)$ & $1.7(1)$ & $5.9(8)$ & $1.6(2)$ & \\
\hline Experience & $(n=328)$ & $(n=61)$ & $(n=141)$ & $(n=126)$ & $0.011 \S$ \\
\hline Median (IQR) & $10(8-10)$ & $9(7-10)$ & $10(8-10)$ & $10(8-10)$ & \\
\hline Duration of wait (minutes) & $(n=334)$ & $(n=62)$ & $(n=141)$ & $(n=131)$ & $<0.001 \S$ \\
\hline Median (IQR) & $5(1-30)$ & $47.5(30-86.25)$ & $15(5-30)$ & $1(0-2)$ & \\
\hline Duration of consultation (minutes) & $(n=332)$ & $(n=61)$ & $(n=141)$ & $(n=130)$ & $<0.001 \S$ \\
\hline Median (IQR) & $5(2-10)$ & $10(5-27.5)$ & $10(5-10)$ & $2(1-3)$ & \\
\hline MISS 21 items & $(n=268)$ & $(n=63)$ & $(n=141)$ & $(n=101)$ & \\
\hline Total score: mean (SD) & $5.27(0.97)$ & $5.39(0.94)$ & $5.70(1.01)$ & $4.75(0.81)$ & $<0.001 \dagger$ \\
\hline Distress relief: mean (SD) & $4.97(1.24)$ & $5.29(1.14)$ & $5.39(1.17)$ & $4.31(1.09)$ & $<0.001 \dagger$ \\
\hline Communication: mean (SD) & $5.50(1.19)$ & $5.43(1.24)$ & $5.89(1.16)$ & $5.05(1.05)$ & $<0.001 \dagger$ \\
\hline Rapport: mean (SD) & $5.38(1.06)$ & $5.48(1.02)$ & $5.83(1.32)$ & $4.83(0.91)$ & $<0.001 \dagger$ \\
\hline Compliance intent: mean (SD) & $5.40(1.10)$ & $5.41(1.18)$ & $5.69(1.05)$ & $5.04(1.02)$ & $<0.001 \dagger$ \\
\hline \multicolumn{6}{|l|}{$\begin{array}{l}{ }^{*} \text { Pearson } \chi^{2} \text {. } \\
\text { †ANOVA. } \\
\text { †For } 2 \times 2 \text { where neither and dissatisfied are combined. } \\
\text { §Kruskal-Wallis test. } \\
\text { In is for total score. }\end{array}$} \\
\hline
\end{tabular}


Table 3 Logistic regression to assess factors associated with symptom resolution

\begin{tabular}{|c|c|c|c|c|c|c|}
\hline \multirow[b]{2}{*}{ Per cent $(\mathrm{n})$ within variable } & \multirow{2}{*}{$\begin{array}{l}\text { Resolved } \\
\% \text { (n) }\end{array}$} & \multirow{2}{*}{$\begin{array}{l}\text { Not resolved } \\
\%(n)\end{array}$} & \multicolumn{2}{|c|}{ Unadjusted } & \multicolumn{2}{|c|}{ Adjusted } \\
\hline & & & $\overline{\mathrm{OR}}$ & $95 \% \mathrm{Cl}$ & OR & $95 \% \mathrm{Cl}$ \\
\hline \multicolumn{7}{|l|}{ Site } \\
\hline Pharmacy & $44.3(43)$ & $55.7(54)$ & 1.00 & & 1.00 & \\
\hline General practice & $35.7(41)$ & $64.3(74)$ & 0.70 & (0.40 to 1.21$)$ & 0.72 & $(0.35$ to 1.49$)$ \\
\hline ED & $37.3(19)$ & $62.7(32)$ & 0.75 & (0.37 to 1.49$)$ & 0.97 & (0.37 to 2.57$)$ \\
\hline \multicolumn{7}{|l|}{ Presenting symptoms } \\
\hline Musculoskeletal aches/pains & $22.9(27)$ & $77.1(91)$ & 1.00 & & 1.00 & \\
\hline Eye pain/discomfort & $73.3(22)$ & $26.7(8)$ & 9.27 & (3.71 to 23.2 ) & 7.96 & (2.93 to 21.6$)$ \\
\hline Stomach upset & $63.6(7)$ & $36.4(4)$ & 5.90 & (1.61 to 21.7 ) & 7.01 & (1.73 to 28.5$)$ \\
\hline Upper respiratory tract & $50.0(41)$ & $50.0(41)$ & 3.37 & (1.83 to 6.20$)$ & 2.80 & (1.28 to 6.14$)$ \\
\hline Multiple symptoms & $27.3(6)$ & $72.7(16)$ & 1.26 & (0.45 to 3.55$)$ & 1.70 & (0.48 to 6.14$)$ \\
\hline \multicolumn{7}{|l|}{ Gender } \\
\hline Male & $43.5(40)$ & $56.5(52)$ & 1.00 & & 1.00 & \\
\hline Female & $37.3(63)$ & $62.7(106)$ & 0.77 & (0.46 to 1.30$)$ & 0.67 & (0.35 to 1.27$)$ \\
\hline \multicolumn{7}{|l|}{ Employment } \\
\hline Employed full time & $42.0(63)$ & $58.0(87)$ & 1.00 & & & \\
\hline Retired & $33.8(24)$ & $66.2(47)$ & 0.71 & (0.39 to 1.27$)$ & & \\
\hline Other (including student, unemployed) & $40.0(16)$ & $60.0(24)$ & 0.92 & (0.45 to 1.87$)$ & & \\
\hline \multicolumn{7}{|l|}{ Marital status } \\
\hline Single & $50.0(25)$ & $50.0(25)$ & 1.00 & & & \\
\hline Married/living with partner & $37.4(65)$ & $62.6(109)$ & 0.60 & (0.32 to 1.12$)$ & & \\
\hline Divorced/separated/widowed & $35.1(13)$ & $64.9(24)$ & 0.54 & $(0.23$ to 1.30$)$ & & \\
\hline \multicolumn{7}{|l|}{ Live alone } \\
\hline Yes & $40.1(83)$ & $64.2(34)$ & 0.84 & (0.45 to 1.56$)$ & & \\
\hline No & $35.8(19)$ & 59.9 (124) & 1.00 & & & \\
\hline \multicolumn{7}{|l|}{ First time had symptoms } \\
\hline Yes & $41.4(41)$ & $58.6(58)$ & 1.18 & (0.71 to 1.97$)$ & & \\
\hline No & $37.4(61)$ & 62.6 (102) & 1.00 & & & \\
\hline \multicolumn{7}{|l|}{ How serious are the symptoms? } \\
\hline Not serious at all & $49.0(48)$ & $51.0(50)$ & 1.00 & & 1.00 & \\
\hline Somewhat/very serious & $33.3(53)$ & $66.7(106)$ & 0.52 & (0.31 to 0.87$)$ & 0.57 & $(0.28$ to 1.27$)$ \\
\hline \multicolumn{7}{|l|}{ Belief on required } \\
\hline Within $24 \mathrm{~h}$ & $48.8(60)$ & $51.2(63)$ & 1.00 & & 1.00 & \\
\hline \multicolumn{7}{|l|}{ Time of visit } \\
\hline $24 \mathrm{~h}$ or more & $29.6(40)$ & $70.4(95)$ & 0.44 & $(0.27$ to 0.74$)$ & 0.42 & $(0.23$ to 0.78$)$ \\
\hline \multicolumn{7}{|l|}{ How long had symptoms? } \\
\hline Median (IQR) days & $3(1-7)$ & $14(3.5-56)$ & 0.99 & (0.99 to 1.00$)$ & 0.99 & $(0.99$ to 1.00$)$ \\
\hline \multicolumn{7}{|l|}{ Baseline EQ-VAS } \\
\hline Mean (SD) & $73.2(17.4)$ & $66.3(20.6)$ & 1.02 & (1.01 to 1.03$)$ & 1.01 & (0.99 to 1.02$)$ \\
\hline \multicolumn{7}{|l|}{ Age (years) } \\
\hline Mean (SD) & $49.8(17.5)$ & $53.7(15.9)$ & 0.99 & $(0.97$ to 1.00$)$ & 0.99 & $(0.97$ to 1.00$)$ \\
\hline
\end{tabular}

pharmacies $(87.9 \%)$ compared with general practice $(61.7 \%)$ or ED $(31.1 \%)$. A higher proportion of participants was supplied other items (eg, dressings) in ED $(37.3 \%)$ compared with being sold or supplied in pharmacy $(7.7 \%)$ or prescribed by general practice $(2.2 \%$; table 2).

Satisfaction was assessed using two measures: MISS-2 $1^{10}$ and global (5-point) scale (very satisfied/very dissatisfied). MISS-21 total and component scores differed significantly $(p<0.001)$ across settings and were consistently highest in general practice and lowest in pharmacy (table 2). While higher satisfaction scores were achieved using the global measure in pharmacy $(68.5 \%)$ and general practice $(66.2 \%)$ compared with
ED $(53.3 \%)$, these were not significant $(\mathrm{p}=0.115$; table 2). The duration of wait for the index consultation was significantly longer $(p<0.001)$ in ED compared with other sites and could have influenced satisfaction ratings (table 2).

Secondary outcomes at follow-up: QoL

QoL measured by the EQ-5D visual analogue scale (EQ-VAS) at follow-up was highest for those who had visited a pharmacy, but differences between sites were not statistically significant (table 2). Participants who visited a pharmacy had the greatest observed change in EQ-VAS compared with baseline (table 2), but this was not statistically different $(p=0.726)$. Employment status 
and participants' perceived seriousness of their symptoms were significant predictors of QoL (EQ-VAS) at follow-up in the univariate analysis (table 4). Only baseline EQ-VAS score and employment status were found to be independent predictors of follow-up QoL in the adjusted linear regression model which includes the a priori variables (table 4). Individuals with higher baseline QoL had higher follow-up QoL and those whose employment status was 'other' (ie, student, unemployed) showed lower QoL at follow-up compared with employed participants. In the adjusted model, neither site of presentation nor presenting symptoms were independent predictors of QoL, although the lower QoL for general practice was of borderline significance $(\mathrm{p}=0.051)$.

\section{Subgroup analysis}

Combining all ailments, no significant difference in proportion resolved was found between sites (table 2). However, as table 5 indicates for musculoskeletal aches and pains only, resolution was higher for ED (31.0\%) and pharmacy $(28.6 \%)$ than for general practice $(9.8 \%$; $\mathrm{p}=0.045$ ). No difference in the proportion resolved was found for sore throat/cough cold with $51.2 \%$ resolving from pharmacy and $48.8 \%$ from general practice $(\mathrm{p}=0.825)$. There were insufficient numbers to compare for eye pain/discomfort or for stomach upset (table 5).

Individuals whose symptoms had resolved had significantly higher QoL at follow-up than for those with unresolved symptoms (table 6). Symptom resolution was also significantly associated with participant satisfaction measured by the MISS-21 (table 6) and this was driven mainly by participants recruited from general practices.

In terms of reconsultation following the index consultation, subsequent visits to general practice or pharmacies were not significantly different for site of presentation (data not presented: available from author). Participants whose index consultation occurred in ED compared with other sites were significantly more likely to return to ED within the following 2 weeks.

\section{Cost-related outcomes}

The mean (95\% CI) overall cost (sum of total initial consultation cost and 2-week follow-up costs) was highest in ED $£ 147.09$ ( $£ 125.32$ to $£ 168.85, \mathrm{n}=48$ ) compared with $£ 82.34$ ( $£ 63.10$ to $£ 101.58, \mathrm{n}=115$ ) and $£ 29.30$ ( $£ 21.60$ to $£ 37.00, \mathrm{n}=95$ ) in general practice and pharmacy, respectively. The mean follow-up QALY score was estimated to be highest for pharmacy participants (a score was estimated for $67.9 \%$ of participants, $n=91$ ), compared with those in general practice $(63.0 \%, \mathrm{n}=102)$ and $\mathrm{ED}(55.6 \%, \mathrm{n}=45)$, although this could partially be accounted for by the fact that they had the highest mean score at the initial visit (this is adjusted for in the subsequent regression analysis).

The number of respondents contributing to the mean incremental cost analysis was 44/81 (54.3\%) for ED, $108 / 162(66.7 \%)$ for general practice and 91/134 $(67.9 \%)$ for pharmacy; these rates did not differ significantly across the three settings $(p=0.10)$. After adjusting for age, gender, baseline EQ-5D-3L score and type of ailment, the mean cost was estimated to be lowest for pharmacy participants; mean $(95 \% \mathrm{CI})$ incremental costs (compared with pharmacy) were estimated to be $£ 57.04$ ( $£ 34.95$ to $£ 79.12$; general practice; $\mathrm{p}<0.001$ ) and $£ 113.63$ ( $£ 81.78$ to $£ 145.47$; ED; $\mathrm{p}<0.001$ ). After adjusting for age, gender, baseline EQ-5D-3L utility score, type of ailment, employment status and whether it was the first time they had experienced the illness, the mean QALY score was estimated to be highest for pharmacy participants, though there were no significant differences between or across settings. The mean (95\% CI) incremental QALY gain for pharmacy participants compared with general practice and ED participants was estimated to be 0.001 (0.000 to 0.002$)$ and 0.001 ( -0.001 to 0.002), respectively. Looking at costs and effects together, as pharmacy was estimated to be less costly and as effective (in terms of symptom resolution) compared with ED and general practice, it can be said to dominate both of these options. It should however be noted that there were no significant differences in outcomes between any of the groups.

\section{Triggers for seeking care}

The main predefined triggers for choice of site were location and convenience of not having to travel too far (table 7). For participants who visited ED, the fact that they would have to wait longer for an appointment with a general practice ranked highly (rank 2, 37\%). Those visiting a pharmacy felt their symptoms were not serious enough to consult a doctor (rank 3,51.5\%), while those visiting the general practice, $63.6 \%$ (rank 2) felt their symptoms were not serious enough for ED. The triggers differed across sites and although "site in a convenient location' was a popular choice for participants recruited from all three types of site, it was more common in pharmacy $(79.1 \%, \mathrm{n}=106)$. 'Knowing the staff' was given as a reason in a higher proportion $(45.7 \%, \mathrm{n}=74)$ of those visiting the general practice compared with other settings. Participants were more likely to seek care from ED if they had not experienced the symptom previously or if the symptom was of recent onset (table 1). The former suggests that previous experience may affect decision-making regarding health-seeking behaviour.

\section{DISCUSSION}

\section{Principal findings}

This is the first study to compare the health-related and cost-related outcomes associated with the management of minor ailments in these three healthcare settings: EDs, general practices and community pharmacies. Symptom resolution and mean improvement in QoL was similar across participants from all settings suggesting equivalence in health outcome. While the absolute rate of symptom resolution appeared to be better in pharmacy, the overall lower than anticipated resolution rates 
Table 4 Factors affecting QoL (EQ-VAS) at follow-up

\begin{tabular}{|c|c|c|c|c|c|}
\hline \multirow[b]{2}{*}{ Per cent $(n)$ within variable } & \multirow[b]{2}{*}{ Mean (SD) } & \multirow{2}{*}{$\begin{array}{l}\text { Univariate } \\
\text { p Value }\end{array}$} & \multicolumn{3}{|c|}{ Adjusted linear regression $\left(\mathrm{R}^{2}=33 \%\right)$} \\
\hline & & & Coefficient & $95 \% \mathrm{Cl}$ & p Value \\
\hline Constant term & & & 54.0 & (38.1 to 56.6 ) & \\
\hline \multicolumn{6}{|l|}{ Site } \\
\hline Pharmacy & $80.9(15.3)$ & $0.054^{*}$ & Ref & & \\
\hline General practice & 75.5 (19.5) & & -4.58 & $(-9.19$ to 0.02$)$ & 0.051 \\
\hline ED & $74.8(19.6)$ & & -3.61 & $(-9.72$ to 2.49$)$ & 0.245 \\
\hline \multicolumn{6}{|l|}{ Ailment } \\
\hline Musculoskeletal aches/pains & $73.1(19.4)$ & $0.005 \dagger$ & Ref & & \\
\hline Eye pain/discomfort & $81.2(16.5)$ & & 1.56 & $(-4.66$ to 7.79$)$ & 0.622 \\
\hline Stomach upset & $84.7(11.2)$ & & 7.22 & $(-1.92$ to 16.4$)$ & 0.121 \\
\hline URT & $81.8(16.5)$ & & 4.61 & $(-0.38$ to 9.60$)$ & 0.070 \\
\hline Multiple ailments & $74.8(18.4)$ & & 5.33 & $(-2.65$ to 13.3$)$ & 0.189 \\
\hline \multicolumn{6}{|l|}{ Gender } \\
\hline Male & $76.6(18.2)$ & $0.536 \ddagger$ & Ref & & \\
\hline Female & 78.0 (18.2) & & -0.12 & $(-4.25$ to 4.02$)$ & 0.956 \\
\hline \multicolumn{6}{|l|}{ Employment } \\
\hline Employed & $80.6(15.9)$ & $0.010^{*}$ & Ref & & \\
\hline Retired & $74.9(18.7)$ & & -3.28 & (-8.99 to 2.43$)$ & 0.259 \\
\hline Other (including student, unemployed) & $70.6(23.1)$ & & -8.27 & $(-14.0$ to -2.55$)$ & 0.005 \\
\hline \multicolumn{6}{|l|}{ Marital status } \\
\hline Single & $78.9(17.1)$ & $0.736 \dagger$ & & & \\
\hline Married/living with partner & 77.5 (18.6) & & & & \\
\hline Divorced/separated/widowed & $75.8(18.1)$ & & & & \\
\hline \multicolumn{6}{|l|}{ Live alone } \\
\hline Yes & $77.6(18.7)$ & $0.898 \ddagger$ & & & \\
\hline No & $77.2(16.6)$ & & & & \\
\hline \multicolumn{6}{|l|}{ First time experienced illness? } \\
\hline Yes & $76.1(18.9)$ & $0.159 \ddagger$ & & & \\
\hline No & $79.4(17.1)$ & & & & \\
\hline \multicolumn{6}{|l|}{ How serious? } \\
\hline Not serious at all & $82.5(14.1)$ & $<0.001 \ddagger$ & Ref & & \\
\hline Somewhat/very serious & 74.7 (19.7) & & -0.55 & $(-5.05$ to 3.96$)$ & 0.811 \\
\hline \multicolumn{6}{|l|}{ Belief on required } \\
\hline Within $24 \mathrm{~h}$ & $79.4(16.9)$ & $0.075 \ddagger$ & & & \\
\hline \multicolumn{6}{|l|}{ Time of visit } \\
\hline $24 \mathrm{~h}$ or more & $\begin{array}{l}75.3(19.3) \\
\text { Correlation }\end{array}$ & & & & \\
\hline \multicolumn{6}{|l|}{ How long had ailment? } \\
\hline (Days) & $-0.262 \S$ & $<0.001$ & -0.001 & $(-0.002$ to 0.000$)$ & 0.163 \\
\hline Baseline EQ-VAS & $0.503 \S$ & $<0.001$ & 0.47 & $(0.37$ to 0.57$)$ & $<0.001$ \\
\hline \multicolumn{6}{|l|}{ Age } \\
\hline (Years) & $-0.059 \S$ & 0.343 & -0.11 & $(-0.27$ to 0.5$)$ & 0.181 \\
\hline $\begin{array}{l}\text { Mean (SD) presented as convention for QoL, b } \\
\text { *Kruskal-Wallis test. } \\
\text { †ANOVA. } \\
\text { fIndependent } t \text { test. } \\
\text { §Spearman's rank correlation. } \\
\text { ANOVA, analysis of variance; ED, emergency }\end{array}$ & esting may be & parametric $d$ & dent on other & umptions. & \\
\hline
\end{tabular}

across all settings, together with reduced numbers of participants at follow-up, meant that the study was under-powered to detect whether these differences were statistically significant.

Mean overall costs were significantly lower for pharmacy consultations compared with general practice consultations and with ED consultations which were associated with the highest costs. The results suggest that substantial savings could be achieved if demand on ED and general practice was shifted to the community pharmacy setting. Some caution is needed because the perceived seriousness and urgency with which to seek care, as well as whether the participant had experienced the symptom(s) previously, varied across settings.

Factors which influenced the patients' site of choice were explored using measures of symptom characteristics, as well as predefined triggers previously shown to influence care-seeking behaviour. ${ }^{11}$ In this study, the 
Table 5 Resolution of symptoms by ailment

\begin{tabular}{|c|c|c|c|c|c|}
\hline & $\begin{array}{l}\text { Overall } \\
\% \text { (n) }\end{array}$ & $\begin{array}{l}\text { ED } \\
\%(n)\end{array}$ & $\begin{array}{l}\text { General } \\
\text { practice } \\
\% \text { (n) }\end{array}$ & $\begin{array}{l}\text { Pharmacy } \\
\% \text { (n) }\end{array}$ & p Value \\
\hline \multicolumn{6}{|l|}{ Resolution of symptoms } \\
\hline Musculoskeletal aches and pains & $(n=118)$ & $(n=42)$ & $(n=41)$ & $(n=35)$ & $0.045^{\star}$ \\
\hline Resolved & $22.9(27)$ & $31.0(13)$ & $9.8(4)$ & $28.6(10)$ & \\
\hline Sore throat/cough cold & $(n=82)$ & - & $(n=41)$ & $(n=41)$ & $0.825^{\star}$ \\
\hline Resolved & $50.0(41)$ & - & $48.8(20)$ & $51.2(21)$ & \\
\hline Eye pain/discomfort† & $(n=30)$ & $(n=7)$ & $(n=12)$ & $(n=11)$ & - \\
\hline Resolved & $73.3(22)$ & $71.4(5)$ & $83.3(10)$ & $63.6(7)$ & \\
\hline Stomach upset† & $(n=11)$ & $(n=1)$ & $(n=6)$ & $(n=4)$ & - \\
\hline Resolved & $63.6(7)$ & $100(1)$ & $50.0(3)$ & $75.0(3)$ & \\
\hline
\end{tabular}

majority (64.2\%) of ED users were experiencing the symptom for the first time with a median onset of one day, perceived it to be somewhat/very serious $(83.1 \%)$ and concluded that a visit to a health service provider was needed within $24 \mathrm{~h}$. These characteristics differentiated ED users from general practice and community pharmacy users. Pharmacy users would have either presented with a direct product request or with a symptom presentation. The former scenario could suggest that the user was familiar with the condition for which they required treatment while the latter would suggest a lack of familiarity with treatment options for the symptom. As such, this may have introduced a bias into the types of user seeking care from community pharmacies. The type of presentation (direct product request/symptom presentation) was not documented during the recruitment process. This study relied on each patient's perception and self-reporting of their symptoms and did not include any qualitative exploration of these perceptions and health-seeking behaviour. Evidence is needed regarding the key determinants of patients' healthseeking behaviour for minor ailments in general and from the ED setting in particular because of the high costs associated with this setting. Theory-based studies are needed to identify key modifiable determinants of this behaviour which in turn will lead to the identification of behaviour change strategies to target these determinants.

Convenience of location and shorter travelling distances were the most important predefined triggers for the choice of healthcare setting. Among ED participants, one of the major reasons cited for choosing the ED was the expected longer wait to see a general practitioner (GP), which may support the finding that convenience was a major factor in selecting the site of treatment, or that participants may have thought it was urgent, or both. The use of EDs for non-urgent conditions has been shown to be associated with fewer available primary care providers (not pharmacies) ${ }^{20}$ but the vast majority of participants in the current study were registered with a GP so would have had access to general practice services if they had opted to do so.

The positive association between participant satisfaction and symptom resolution is an important finding. An earlier study of acute minor illness where index visits were made to EDs, family physicians or walk-in clinics, showed patient satisfaction with patient-centred care was associated with reduced reutilisation rates. ${ }^{12}$ Doctors' communication behaviour is a major driver of patient satisfaction with GP consultations. ${ }^{21}$ This emphasises the need for good consultation skills among healthcare professionals. Suboptimal communication during community pharmacy consultations has been highlighted as an area for improvement ${ }^{22} 23$ because it is associated with suboptimal and inappropriate outcomes. ${ }^{23-25}$

Other studies have compared combinations of settings, for example, walk-in clinics versus family practice versus ED, ${ }^{12} 13$ but no previous study included community pharmacy as a comparator. This study did not explore whether the patients were seeking care for reasons other than their minor ailment, however, the promotional materials clearly specified the four ailments of interest and as such, an assumption of this study is that the minor ailment was the primary reason for presentation. The inclusion of different types (independent single outlets, small/large chains) of pharmacies, as well as a variety of locations (urban/rural) of pharmacies and general practices, increased the generalisability of the results. The participants' choice of setting had face validity; symptoms that were perceived as somewhat serious (and therefore might be more worrying and require expert medical attention) presented more often in ED than in other settings. All participating sites were within 25 miles of a major city, therefore the results may not be generalisable to people living in more remote areas. People in these areas may behave differently when managing their symptoms, especially if convenience is a major influencing factor for them too. Both EDs were located in teaching hospitals and as such, the results may not be generalisable to EDs in other types of 


\begin{tabular}{|c|c|c|c|c|c|c|c|c|}
\hline & \multicolumn{2}{|c|}{ ALL } & \multicolumn{2}{|l|}{ ED } & \multicolumn{2}{|c|}{ General practice } & \multicolumn{2}{|c|}{ Pharmacy } \\
\hline & $\bar{n}$ & Mean (SD) & $\bar{n}$ & Mean (SD) & $\bar{n}$ & Mean (SD) & $\mathrm{n}$ & Mean (SD) \\
\hline \multicolumn{9}{|c|}{ EQ-5D-3L utility baseline } \\
\hline Resolved & 101 & $0.74(0.22)$ & 18 & $0.58(0.28)$ & 40 & $0.76(0.22)$ & 43 & $0.79(0.17)$ \\
\hline Not resolved & 148 & $0.64(0.28)$ & 29 & $0.49(0.32)$ & 69 & $0.64(0.26)$ & 50 & $0.73(0.26)$ \\
\hline p Value* & & 0.002 & & 0.362 & & 0.01 & & 0.195 \\
\hline \multicolumn{9}{|c|}{ EQ-5D-3L utility follow-up } \\
\hline Resolved & 99 & $0.93(0.12)$ & 18 & $0.94(0.09)$ & 39 & $0.95(0.10)$ & 42 & $0.91(0.15)$ \\
\hline Not resolved & 150 & $0.71(0.26)$ & 30 & $0.62(0.31)$ & 67 & $0.71(0.27)$ & 35 & $0.77(0.22)$ \\
\hline$p$ Value* & & $<0.001$ & & $<0.001$ & & $<0.001$ & & $<0.001$ \\
\hline \multicolumn{9}{|c|}{ EQ-VAS baseline } \\
\hline Resolved & 103 & $73.2(17.4)$ & 19 & $73.1(18.0)$ & 41 & $72.6(17.8)$ & 43 & $73.7(17.2)$ \\
\hline Not resolved & 158 & $66.3(20.6)$ & 32 & $64.5(24.2)$ & 72 & $64.6(19.8)$ & 54 & $70.0(19.2)$ \\
\hline p Value* & & 0.004 & & 0.187 & & 0.034 & & 0.285 \\
\hline \multicolumn{9}{|c|}{ EQ-VAS follow-up } \\
\hline Resolved & 103 & $86.3(11.6)$ & 19 & $81.5(14.1)$ & 41 & $87.0(10.6)$ & 43 & $87.7(11.1)$ \\
\hline Not resolved & 157 & $71.5(19.4$ & 31 & $70.8(21.6)$ & 73 & $69.0(20.5)$ & 54 & $75.4(16.1)$ \\
\hline \multirow[t]{2}{*}{$\mathrm{p}$ Value* } & & $<0.001$ & & 0.061 & & $<0.001$ & & $<0.001$ \\
\hline & $\mathbf{n}$ & Median (IQR) & $\mathbf{n}$ & Median (IQR) & $\mathbf{n}$ & Median (IQR) & $\mathbf{n}$ & Median (IQR) \\
\hline \multicolumn{9}{|l|}{ Experience } \\
\hline Resolved & 100 & $10(8-10)$ & 18 & $9.5(7.75-10)$ & 41 & $10(9-10)$ & 41 & $10(8-10)$ \\
\hline Not resolved & 155 & $10(8-10)$ & 30 & $9(7.75-10)$ & 73 & $10(8-10)$ & 52 & $10(8.25-10)$ \\
\hline $\mathrm{p}$ Value & & 0.77 & & 0.46 & & 0.958 & & 0.201 \\
\hline \multicolumn{9}{|l|}{ MISS 21 scores } \\
\hline \multicolumn{9}{|l|}{ TOTAL score } \\
\hline Resolved & 90 & $5.38(4.71-6.15)$ & 15 & $5.48(4.95-6.62)$ & 37 & $6.05(5.19-6.69)$ & 38 & $4.74(4.14-5.48)$ \\
\hline Not resolved & 119 & $5.00(4.48-5.90)$ & 26 & $5.31(4.79-6.01)$ & 56 & $5.19(4.81-6.37)$ & 37 & $4.43(4.00-5.02)$ \\
\hline $\mathrm{p}$ Value† & & 0.047 & & 0.291 & & 0.015 & & 0.116 \\
\hline \multicolumn{9}{|l|}{ Distress } \\
\hline Resolved & 90 & $5.00(4.17-6.00)$ & 15 & $5.50(5.00-7.00)$ & 37 & $6.00(5.00-7.00)$ & 38 & $4.17(4.00,5.00)$ \\
\hline Not resolved & 119 & $4.67(4.0-5.67)$ & 26 & $5.33(4.46-6.08)$ & 56 & $5.19(4.21-6.0)$ & 37 & $4.00(3.92,4.50)$ \\
\hline $\mathrm{p}$ Value† & & 0.009 & & 0.074 & & 0.004 & & 0.046 \\
\hline \multicolumn{9}{|l|}{ Communication } \\
\hline Resolved & 90 & $5.00(4.75-6.75)$ & 15 & $5.50(5.00-7.00)$ & 37 & $6.50(5.25-7.00)$ & 38 & $5.00(4.25,6.25)$ \\
\hline Not resolved & 119 & $5.00(4.50-6.25)$ & 26 & $5.13(4.69-6.50)$ & 56 & $5.50(5.00-7.00)$ & 37 & $4.75(4.13,5.50)$ \\
\hline $\mathrm{p}$ Valuet & & 0.161 & & 0.348 & & 0.153 & & 0.206 \\
\hline \multicolumn{9}{|l|}{ Rapport } \\
\hline Resolved & 90 & $5.38(4.50-6.28)$ & 15 & $5.75(4.75-6.75)$ & 37 & $6.00(5.38-6.88)$ & 38 & $4.56(4.22,5.38)$ \\
\hline Not resolved & 119 & $5.00(4.75-6.00)$ & 26 & $5.44(4.84-6.31)$ & 56 & $5.25(4.88-6.88)$ & 37 & $4.75(4.00,5.06)$ \\
\hline $\mathrm{p}$ Valuet & & 0.834 & & 0.668 & & 0.403 & & 0.5 \\
\hline \multicolumn{9}{|c|}{ Compliance intent } \\
\hline Resolved & 90 & $5.50(4.92-6.67)$ & 15 & $6.00(5.00-6.67)$ & 37 & $6.00(5.00-7.00)$ & 38 & $5.00(4.00,6.00)$ \\
\hline Not resolved & 119 & $5.00(4.33-6.00)$ & 26 & $5.17(4.33-6.00)$ & 56 & $5.33(5.00-6.33)$ & 37 & $4.43(4.00,5.00)$ \\
\hline $\mathrm{p}$ Value & & 0.057 & & 0.119 & & 0.12 & & 0.136 \\
\hline
\end{tabular}


Table 7 Triggers for choice of site

\begin{tabular}{|c|c|c|c|c|c|c|c|}
\hline \multirow[b]{2}{*}{ Per cent $(n)$ unless specified } & \multicolumn{2}{|l|}{ ED } & \multicolumn{2}{|c|}{ General practice } & \multicolumn{2}{|l|}{ Pharmacy } & \multirow[b]{2}{*}{ p Value* } \\
\hline & $(\mathrm{N}=81)$ & Rank & $(\mathrm{N}=162)$ & Rank & $(\mathrm{N}=134)$ & Rank & \\
\hline \multicolumn{8}{|l|}{ Location and time } \\
\hline The 'site 'is in a location convenient for me $\dagger$ & $51.9(42)$ & 1 & $69.1(112)$ & 1 & $79.1(106)$ & 1 & $<0.001$ \\
\hline Did not have to travel far to visit the 'site' $\dagger$ & $34.6(28)$ & 3 & $51.2(83)$ & 3 & $57.4(77)$ & 2 & $<0.001$ \\
\hline I was already visiting 'site 'for different reason† & $2.5(2)$ & 17 & $6.8(11)$ & 16 & $35.8(48)$ & 7 & $<0.001$ \\
\hline It was not convenient to go to the chemist & $1.2(1)$ & 19 & $1.9(3)$ & 18 & NA & - & Low coun \\
\hline It was not convenient to go to the GP & $13.6(11)$ & 11 & NA & - & $15.7(21)$ & 17 & 0.837 \\
\hline $\begin{array}{l}\text { I can get treatment/advice at 'site 'without } \\
\text { appointment }\end{array}$ & NR & - & NA & - & $36.6(49)$ & 6 & \\
\hline \multicolumn{8}{|l|}{ Knowing the staff } \\
\hline I know the 'staff 'heret & $1.2(1)$ & 19 & $45.7(74)$ & 5 & $26.9(36)$ & 11 & $<0.001$ \\
\hline I feel the 'staff 'here know me $\dagger$ & $3.7(3)$ & 14 & $30.9(50)$ & 10 & $19.4(26)$ & 14 & $<0.001$ \\
\hline $\begin{array}{l}\text { I am comfortable discussing my illness with 'staff' } \\
\text { here }\end{array}$ & $23.5(19)$ & 8 & $51.2(83)$ & 3 & $47.8(64)$ & 4 & $<0.001$ \\
\hline 'staff' in 'site' in general know about this illness $\dagger$ & $25.9(21)$ & 7 & $41.4(67)$ & 6 & $30.6(41)$ & 8.5 & 0.037 \\
\hline $\begin{array}{l}\text { I have previously successfully used 'site' to treat this } \\
\text { illness } \dagger\end{array}$ & $16.0(13)$ & 9 & $32.1(52)$ & 9 & $38.1(51)$ & 5 & 0.003 \\
\hline I could discuss my illness with 'staff' in private† & $13.6(11)$ & 11 & $39.5(64)$ & 7 & $21.6(29)$ & 12 & $<0.001$ \\
\hline $\begin{array}{l}\text { I needed information about illness from 'staff' at } \\
\text { 'site' } \dagger\end{array}$ & $34.6(28)$ & 3 & $37.0(60)$ & 8 & $20.1(28)$ & 13 & 0.009 \\
\hline \multicolumn{8}{|l|}{ Other options } \\
\hline My illness was too serious to go to GP & $30.9(25)$ & 5 & NA & - & NR & - & - \\
\hline My illness was too serious to go to chemist & $27.2(22)$ & 6 & NR & - & NA & - & - \\
\hline My illness was not serious enough for ED & NA & - & $63.6(103)$ & 2 & NR & - & - \\
\hline My illness was not serious enough for a doctor & NA & - & NA & - & $51.5(69)$ & 3 & - \\
\hline I did not want to waste the doctor's time & NR & - & NA & - & $30.6(41)$ & 8.5 & - \\
\hline $\begin{array}{l}\text { I previously consulted GP about illness, but it is not } \\
\text { better }\end{array}$ & $16.0(13)$ & 9 & NA & - & $18.7(25)$ & 15 & 0.775 \\
\hline $\begin{array}{l}\text { I previously consulted pharmacy about illness, but it } \\
\text { is not better }\end{array}$ & $3.7(3)$ & 14 & $9.9(16)$ & 15 & NA & - & 0.158 \\
\hline I would have to wait longer for appointment at GP & $37.0(30)$ & 2 & NA & - & $29.9(40)$ & 10 & $<0.001$ \\
\hline $\begin{array}{l}\text { I would have to wait longer for appointment at other } \\
\text { services }\end{array}$ & NR & - & $14.8(24)$ & 13 & NR & - & - \\
\hline I prefer not to go to ED & NA & & $28.4(46)$ & 11 & NR & - & - \\
\hline I prefer not to go to GP & $3.7(3)$ & 14 & NA & - & $11.2(15)$ & 19 & 0.097 \\
\hline I prefer not to go to a chemist & $4.9(4)$ & 13 & $16.7(27)$ & 12 & NA & - & 0.019 \\
\hline \multicolumn{8}{|l|}{ Cost of treatment } \\
\hline It was cheaper to visit than other services $†$ & $1.2(1)$ & 19 & $6.8(11)$ & 16 & $17.9(24)$ & 16 & $<0.001$ \\
\hline Treatment/advice was cheaper for my illness $\dagger$ & $1.2(1)$ & 19 & $11.7(19)$ & 14 & $14.9(20)$ & 18 & 0.006 \\
\hline \multicolumn{8}{|l|}{ Advice/referral } \\
\hline $\begin{array}{l}\text { I came to get advice/treatment that I had seen in } \\
\text { mediat }\end{array}$ & $2.5(2)$ & 17 & $0(0)$ & 19 & $1.5(2)$ & 20 & unts \\
\hline
\end{tabular}

${ }^{*}$ Pearson $\chi^{2}$ test.

†ltem recorded on for all sites.

ED, emergency department; GP, general practitioner; NA, not applicable for that site; NR, item not recorded for that site version.

hospital. There was slight under-recruitment from community pharmacies. This study used a cohort design rather than randomised controlled trial design because the latter was an impractical option to address the objectives of this study. Previous research identified that perceptions of service quality influence care seeking from $\mathrm{EDs}^{26}$ and that competence and credibility of staff was important and more highly rated for staff in EDs compared with general practices. The current study did not include measures of quality perception nor assess participants' perceptions of staff credibility or competence.

\section{Meaning of the study}

Despite the limitations of this study in terms of sample size, reliance on self-reported data and focusing on selected common symptoms, the results provide evidence of the value of community pharmacy as a resource from the management of these types of conditions. The Keogh report highlighted the 'intense, growing and unsustainable pressure' being placed on EDs and the need to direct demand outside of hospitals for conditions that may be "urgent but non-life threatening. ${ }^{3}$ The same report identified that community 
pharmacies are an 'under-utilised resource's which could contribute substantially to reduce the burden on other healthcare providers. Self-care is the preferred method of managing minor illness for many patients ${ }^{27}$ and the need to support patients with self-care has been acknowledged as a priority. ${ }^{3}$ The results of this cohort study suggest that patients with minor ailments presenting in community pharmacies will have similar health outcomes to those in general practice and ED, but that the costs will be substantially lower with pharmacymanaged consultations. Redirection of demand needs to address accessibility, affordability, availability and most importantly, safety of care. ${ }^{28}$ Earlier interventions to promote the self-management of minor illness have had limited effect; ${ }^{29}$ few, if any, have adopted a theory-based approach to exploring behaviour and promoting behaviour change.

\section{Unanswered questions and future research}

Convenience of location was a major influence on health-seeking behaviour for the conditions included in this study. The evidence for co-locating community pharmacies with EDs and general practices is currently lacking, but the co-location of walk-in clinics within EDs failed to influence patient satisfaction, preference or choice. $^{30}$ Patients' perceptions that obtaining a GP appointment would 'take too long' is one which requires further investigation to determine what urgent care arrangements should be available within general practices to manage acute conditions perceived to be serious or in need of immediate attention. The substantially lower costs associated with pharmacy management of these ailments, combined with similar health outcomes across settings, suggest there is an urgent need to identify effective interventions to promote the use of community pharmacies for the management of minor ailments. These interventions should be evidence-based and underpinned by behaviour change theory to maximise their effectiveness and reproducibility. Further research is warranted and planned regarding patients' decisionmaking processes for choosing their care provider when managing minor ailments.

\section{Author affiliations}

${ }^{1}$ Division of Applied Health Sciences, Centre of Academic Primary Care, University of Aberdeen, Aberdeen, UK

${ }^{2}$ Emergency Department, Aberdeen Royal Infirmary, Aberdeen, UK

${ }^{3}$ Health Economics Group, Norwich Medical School, Norwich, UK

${ }^{4}$ Norwich Medical School, University of East Anglia, Norwich, UK

${ }^{5}$ School of Pharmacy, University of East Anglia, Norwich, UK

\section{Twitter Follow Margaret Watson at @MagsWatson1}

Acknowledgements The authors thank the patients and healthcare providers who participated in this study. They also thank J Burr and J Inch for data collection and $\mathrm{H}$ Riley and $\mathrm{N}$ Clark for their administrative support.

Contributors MCW (guarantor) had the original idea for the study and with JF, CMB, RH, TP, THS and DW designed the trial and formed the investigator group that obtained funding. MCW, RH and SF were responsible for overseeing study implementation and $A B, V M$ and VP were responsible for data collection. SF, THS and GRB conducted the analyses. MCW, SF and GRB drafted the manuscript which was revised by all authors. All researchers were independent of the funders and approved the final version.

Funding This work was supported by the Pharmacy Practice Research Trust which merged with the Pharmaceutical Trust for Educational and Charitable Objects in December 2012 to form Pharmacy Research UK.

Competing interests None.

Patient consent Obtained.

Ethics approval The North of Scotland Research Ethics Committee.

Provenance and peer review Not commissioned; externally peer reviewed.

Data sharing statement No additional data are available.

Open Access This is an Open Access article distributed in accordance with the Creative Commons Attribution Non Commercial (CC BY-NC 4.0) license, which permits others to distribute, remix, adapt, build upon this work noncommercially, and license their derivative works on different terms, provided the original work is properly cited and the use is non-commercial. See: http:// creativecommons.org/licenses/by-nc/4.0/

\section{REFERENCES}

1. The College of Emergency Medicine. The drive for quality. How to achieve safe, sustainable care in our emergency departments? London: The College of Emergency Medicine, 2013.

2. Health and Social Care Information Centre. Focus on accident and emergency. http://www.hscic.gov.uk/catalogue/PUB13040/acciemer-focu-on-2013-rep-V2.pdf. Updated 2013(accessed 26 Mar 2014).

3. NHS England. Transforming urgent and emergency care services in England. Urgent and emergency care review end of phase 1 report. Appendix 1-Revised Evidence Base from the Urgent and Emergency Care Review. Leeds: NHS England, 2013.

4. Hippisley-Cox J, Vinogradova Y. Trends in consultation rates in general practice 1995/1996 to 2008/9: analysis of the QResearch database. Final report to NHS information Centre and Department of Health. Nottingham: The NHS Information Centre for Health and Social Care, 2009.

5. Watson MC, Holland R, Ferguson J, et al. Community pharmacy management of minor illness (the MINA study). London: Pharmacy Research UK, 2014.

6. Jones R, White R, Armstrong D, et al. Managing acute illnesses: an enquiry into the quality of general practice in England. London: The King's Fund, 2010.

7. The Royal Pharmaceutical Society of Great Britain. RPS e-PIC references on: prescription only medicines reclassified to pharmacy only medicines. London: RPSGB, 2008.

8. Paudyal V, Watson MC, Sach T, et al. Are pharmacy-based minor ailment schemes a substitute for other service providers? A systematic review. Br J Gen Pract 2013;63:e472-81.

9. EuroQol Research Foundation. EQ-5D. http://www.euroqol.org/

10. Meakin R, Weinman J. The 'Medical interview satisfaction scale' (MISS-21) adapted for British general practice. Fam Pract 2002;19:257-63.

11. Porteous T. How do people choose between self-care, supported self-care and GP consultation in minor illness? [PhD thesis]. University of Aberdeen, 2007.

12. Campbell MK, Silver RW, Hoch JS, et al. Re-utilization outcomes and costs of minor acute illness treated at family physician offices, walk-in clinics, and emergency departments. Can Fam Physician 2005;51:82-93.

13. Hutchison B, Østbye T, Barnsley J, et al. Patient satisfaction and quality of care in walk-in clinics, family practices and emergency departments: the Ontario walk-in clinic study. Can Med Assoc J 2003;168:977-83.

14. Brooks R, De Charro F. EuroQol: the current state of play. Health Policy 1996;37:53-72.

15. Schafheutle E, Noyce P, Sheehy C, et al. Evaluation of a pilot scheme to allow patients exempt from prescription charges to consult community pharmacists and receive 'over the counter 'products for minor ailments free of charge under the NHS Direct Supply by community pharmacists. Edinburgh: Scottish Government, 2002.

16. Briggs A, Clark T, Wolstenholme J, et al. Missing...presumed at random: cost-analysis of incomplete data. Health Econ 2003;12:377-92. 
17. Manca A, Hawkins N, Sculpher MJ. Estimating mean QALYs in trial-based cost-effectiveness analysis: the importance of controlling for baseline utility. Health Econ 2005;14:487-96.

18. Drummond MF, Sculpher MJ, Torrance GW, et al. Methods for the economic evaluation of health care programmes. 3rd edn. New York: Oxford University Press, 2005.

19. NICE Guide to the methods of technology appraisal. London: National Institute for Health and Care Excellence, 2013.

20. Mathison DJ, Chamberlain JM, Cowan NM, et al. Primary care spatial density and nonurgent emergency department utilization: a new methodology for evaluating access to care. Acad Pediatr 2013;13:278-85.

21. Paddison $\mathrm{C}$, Abel G, Roland M, et al. Drivers of overall satisfaction with primary care: evidence from the English general practice patient survey. Health Expect 2013. doi:10.1111/hex.12081

22. Which Report. Can you trust your local pharmacy's advice? WHICH? 20 May 2013. http://www.which.co.uk/news/2013/05/can-you-trustyour-local-pharmacys-advice-319886/(accessed 23 Jul 2014).

23. Watson MC, Bond CM, Grimshaw J, et al. Factors predicting the guideline compliant supply (or non-supply) of non-prescription medicines in the community pharmacy setting. $\mathrm{Br}$ Med $\mathrm{J}$ 2006;15:53-7.
24. Watson MC, Walker AE, Bond CM. Community pharmacists' views and beliefs about the treatment of symptoms suggestive of vaginal thrush in community pharmacies. Pharm World Sci 2000;22:130-5.

25. Watson MC, Bond CM, Grimshaw JM, et al. Educational strategies to promote evidence-based community pharmacy practice: a cluster randomized controlled trial (RCT). Fam Pract 2002;19:529-36.

26. Lo S, Mc Kechnie S. Perceptions of service quality and sacrifice in patients with minor medical conditions using emergency care. Int J Clin Pract 2007;61:596-602.

27. Porteous T, Ryan M, Bond CM, et al. Preferences for self-care or professional advice for minor illness: a discrete choice experiment. Br J Gen Pract 2006;56:911-17.

28. Durand AMP, Gentile S, Devictor B, et al. ED patients: how nonurgent are they? Systematic review of the emergency medicine literature. Am J Emerg Med 2011;29:333-45.

29. Little P, Somerville J, Williamson I, et al. Randomised controlled trial of self management leaflets and booklets for minor illness provided by post. BMJ 2001;322:1214-17.

30. Chalder M, Montgomery A, Hollinghurst S, et al. Comparing care at walk-in centres and at accident and emergency departments: an exploration of patient choice, preference and satisfaction. Emerg Med J 2007;24:260-4. 